\title{
Apocrine Chromhidrosis over dorsum of foot: A case report
}

\author{
Arjun Mapare ${ }^{1}$, Vaibhav N. Tapre ${ }^{2}$, Ankit Kumar Khandelwal ${ }^{3}$ \\ ${ }^{1}$ (Asso.Professor,Departmen of Skin \& VD, MIMSR Medical College,Latur,Maharashtra,India) \\ ${ }^{2,3}$ (Resident,Departmen of Skin \& VD, MIMSR Medical College,Latur,Maharashtra,India )
}

\begin{abstract}
Chromohidrosis a rare condition charecterized by the production of coloured sweat, by apocrine gland. Lipofuscin pigment is responsible for the coloured sweat, this pigment is produce in the apocrine gland and its various oxidative states account for the characteristic yellow, green, blue or black secretion. Usually apocrine chromhidrosis is common over face, axillae.Apocrine chromhidrosis over dorsum of foot is extremely rare with few cases reported in literature till date. We report a case of 60 years old female patient who presented to our outpatient department with complaint of bluish coloured secretion coming out of skin over dorsum of foot since 10 days. Based on history and clinical examination provisional diagnosis of apocrine chromohidrosis was made, which is confirmed by laboratory investigation and histopathology. Patient was given capsaicin gel and followed up for 1 month. The rare and unusual occurance of the chromhidrosis prompted us to report the case.
\end{abstract}

Keywords -Chromhidrosis, Apocrine gland, blue sweat.

\section{Introduction}

Chromohidrosis is noted after puberty when the apocrine gland is functional. Apocrine chromhidrosis is charecterised by production of coloured apocrine sweat, The colour is result due to lipofuscin pigment in apocrine secretion and its various oxidative products results in to its various colours.[1],[2]

Lipofuscin is found in higher than normal concentration or a higher than normal state of oxidation in apocrine gland. However the exact etiology of apocrine chromhidrosis is unknown. It is postulated that substance $\mathrm{P}$ act as an important mediator in this process.[2]

\section{Case Report:}

A 60 years old postmenopausal female presented with complaint of bluish secretion appeared over the dorsum of right foot since last 10 days (fig 1). It increases in amount after physical exertion and emotional stress. No any abnormality present over surrounding skin and skin over other body area. After mopping the skin with cotton it get bluish colouration (fig 2).

There is no history of any relavent drug intake (like multivitamin), hormonal replacement therapy (HRT), testosterone creams. No history of any dietary changes, occupational or any external substance application (which rules out pseudochromohidrosis). We did all routine laboratory studies which came out to be normal. We did take local swab for culture which also did not show any growth, so on the basis of history, clinical examination and investigation, a diagnosis of apocrine chromhidrosis was made.

We had started capsaicin gel $(0.1 \%)$ to the patient locally at night time daily, and asked to follow up after 4 weeks. Patient gives history of burning sensation of foot initially after application of the capsaicin gel but afterward she tolerated it well, also she gave history of decreased sweating over the foot.

\section{Figures:}

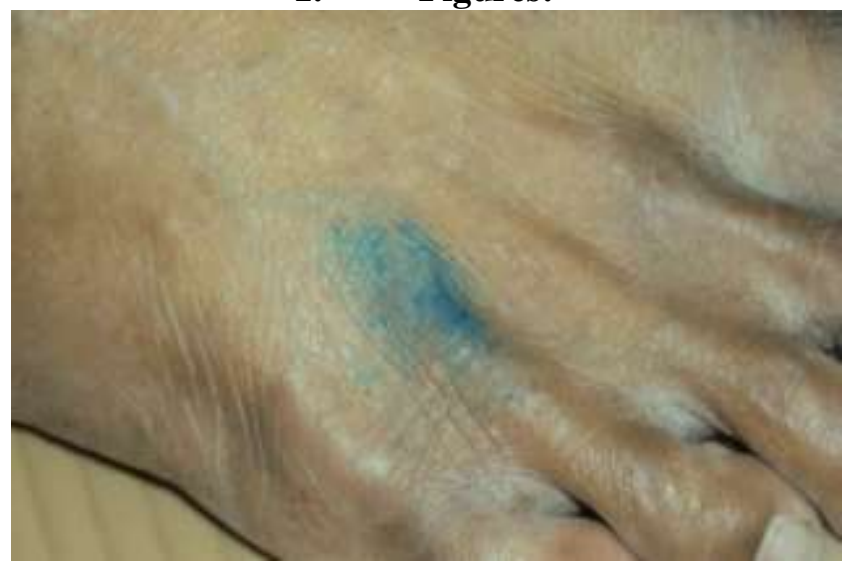

Fig 1: Blue sweat excreted from dorsum of foot 


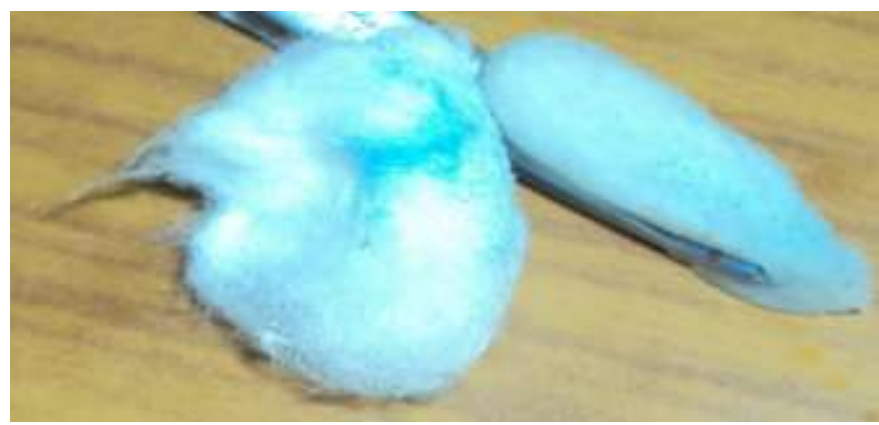

Fig 2: Bluish colouration of cotton swab after mopping dorsum of foot.

\section{Discussion}

Apocrine chromhidrosis is rare disorder with varying clinical presentation. It commonly present over face, areola and axilla,[4],[5],[9] but we report a case having presentation over dorsum of foot which is not reported in literature. On histopathology we can find bluish cytoplasmic granules in the apocrine epithelium lining, which are lipofuscin granules.

It is necessary to differentiate apocrine chromhidrosis from eccrine chromhidrosis and pseudochromhidrosis. Eccrine chromhidrosis occurs due to water soluble pigment excreted from eccrine gland which may occurs after drug intake. Psudochromhidrosis occur as a excretion of colourless sweat mixed with an external chromogens such as dyed clothing, coloured chemicals, or microorganism such as piedria or cornybacterium. [2],[8],[9]

Apocrine chromhidrosis does not cause any problem to person but is affect the psychosocial life of person. So there are various modalities are consider for treatment of chromhidrosis. Topical capsaicin gel is used which deplet the substance $\mathrm{P}$ from the neurons which is a neurotransmitter plays important role in apocrine sweat excretion.[3],[7] Botulinum toxin also considered as treatment option, it also deplete substance $\mathrm{P}$ from neurons.[6]

\section{Conclusion}

Apocrine chromhidrosis is rare disorder mainly present over axilla and face, but it can be present over other body surface area like dorsum of foot. And can be treated with topical capsaicin.

\section{References:}

[1] Coulson IH. Disorders of sweat glands. In : Burns T, Breathnach S, Cox N, Griffiths C. editors. Rook/ Wilkinson/ Ebling Textbook of dermatology. 7h ed. Blackwell Science: Oxford; 1998. p. 45.22-45.33.

[2] Daoud MS, Dicken CH. Disorders of Apocrine sweat glands. In: Freedberg IM, Eisen AZ, Wolff K, Austen KF, Goldsmith LA, Katz SI, editors. Fitzpatrick's Dermatology in general medicine. 6th ed. Mc Graw-Hill: New York; 2003. p. 708-9

[3] Mark JR Jr. Treatment of apocrine chromhidrosis with topical capsaicin. J Am Acad Dermatol 1989;21:418-420.

[4] Saff DM, Owens R,Kahn TA. Apocrine chromhidrosis involving the areola in a 15 yr. Old amateur figure skater.Pediat Dermatal 1995;12:48-50 Griffith JR. Isolated areolar apocrine chronhidrosis. Pediatrics 2005;115:e 239-41.

[5] Matarasso, SL. Treatment of facial chromhidrosis with botulinum toxin type A. J Am Acad Dermatol 2005; 52 (1): 89-91.

[6] Gandhi V,Vij A,Bhattacharya SN. Apocrine chromhidrosis localized to the areola in an Indian treated with topical capsaicin .IJDVL 2006;72( 5):382-383.

[7] Hill S, Duffill M, Lamont D, Rademaker M, Yung A. Pseudochromhidrosis: blue discolouration of the head and neck. Australas J Dermatol. Nov 2007; 48(4):239-41.

[8] Tato BP, Martinez EZ, Albisna BS, Gonzalez YCP, Olabarrieta IP , Escobedo SM,etal.Facial and axillary apocrine chromhidrosis.Dermatol online Journal 2012;18(3):13 